\title{
Comunicación alternativa y comunitaria. La conformación del campo en Europa y el diálogo con América Latina
}

Alternative and community communication. The shaping of the field in Europe and the dialogue with Latin America

Comunicação alternativa e cominitária. A conformação do campo na Europa e o diálogo com a América Latina

\author{
Alejandro BARRANQUERO \\ Universidad Carlos III de Madrid / abarranq@hum.uc3m.es \\ Emiliano TRERÉ \\ Universidad de Cardiff / TrereE@cardiff.ac.uk
}

Chasqui. Revista Latinoamericana de Comunicación

N. ${ }^{\circ}$ 146, abril-julio 2021 (Sección Monográfico, pp. 159-182)

ISSN 1390-1079 / e-ISSN 1390-924X

Ecuador: CIESPAL

Recibido:08-12-2020/Aprobado:05-02-2021 


\title{
Resumen
}

En el siguiente artículo se hace un balance de la historia y actualidad del campo de la comunicación alternativa y comunitaria en el contexto europeo y estadounidense y se compara dicha tradición con los desarrollos latinoamericanos con el fin de establecer semejanzas y discontinuidades. Apoyados en un marco de investigación comparada y revisión crítica documental, señalamos la necesidad de tender puentes entre dichas comunidades académicas de cara a fortalecer el diálogo interdisciplinar e interregional.

Palabras clave: comunicación alternativa; medios comunitarios; movimientos sociales; comunicación para el cambio social; ciudadanía

\begin{abstract}
This article maps the main historical traditions and contemporary debates in the field of alternative and community communication in the European and US contexts, and compares these research frameworks with Latin American theory with the aim of finding similarities and differences. Based on comparative research and critical literature review, this work raises the need for building bridges between European research and the long tradition of Latin American studies in order to strengthen an interdisciplinary and interregional dialogue.
\end{abstract}

Key words: alternative communication; community media; social movements; communication for social change; citizenship

\section{Resumo}

O artigo seguinte faz um balanço da história e do presente do campo da comunicação alternativa e comunitária no contexto europeu e americano e compara esta tradição com os desenvolvimentos latino-americanos, a fim de estabelecer semelhanças e descontinuidades. Com base num quadro de investigação comparativa e de revisão documental crítica, salientamos a necessidade de construir pontes entre estas comunidades académicas a fim de reforçar o diálogo interdisciplinar e inter-regional.

Palavras-chave: comunicação alternativa; meios de comunicação comunitários; movimentos sociais; comunicação para a mudança social; cidadania 


\section{Introducción}

Partimos de la premisa de que la relación entre comunicación, alternatividad y transformaciones sociales ha sido abordada desde distintos enfoques teóricos —ej. Estudios Culturales, Economía Política, Comunicación para el Desarrollo, etc. - y distintos lugares de enunciación: ej. Europa frente a Latinoamérica ${ }^{1}$. Dichas perspectivas han establecido escasos diálogos entre sí, lo que provoca la conformación de tradiciones teóricas distintas y bastante autorreferenciales en sus inspiraciones teóricas. La ausencia de puentes tampoco facilita una comprensión más integral del objeto de estudio, a pesar de que la comunicación alternativa debe abordarse desde la singularidad de los contextos locales y nunca desde marcos universales que obvien la extrema diversidad de la práctica de los movimientos sociales.

Por otro lado, son escasos los trabajos que han avanzado en la línea de la investigación comparada, entendida como el desarrollo de enfoques transculturales que ayudan a comparar macrounidades de conocimiento con relación a regiones, áreas lingüísticas, etc. - , más allá de las fronteras de los Estados-nación (Hepp \& Couldry, 2009). Esta tarea ha tenido avances sustanciales en el campo más amplio de la comunicación para el desarrollo y el cambio social (ej. Servaes, 1999; Manyozo, 2012). Sin embargo, los intentos en el ámbito de la comunicación alternativa y ciudadana se han limitado a readers y monográficos que relacionan experiencias y estudios de caso (Downing, 2010; Atton, 2015) o incluso conceptos (Bakert, Blaagaard, Jones, \& Pérez-González, 2020), pero que raramente establecen conexiones o diálogos entre comunidades lingüísticas o culturales.

Elpresenteensayobasasusargumentosenlatécnicadelarevisióndocumental no sistemática (Grant \& Booth, 2009) de textos clásicos y contemporáneos de referencia en el campo, muchos de los cuales no se citan por ausencia de espacio. Para la selección crítica de los títulos y debates, se han realizado indagaciones en los buscadores especializados Google Academic y Google Books, además de en la base de datos de revistas científicas Scopus. Se han empleado combinaciones de los términos “medios" y “comunicación” con los descriptores propios del área (comunitario, alternativo, popular, radical, ciudadano, etc.) tanto en español como en cuatro idiomas relevantes en la tradición científica europea: inglés, francés, italiano y alemán. Este ejercicio meta-analítico (Gurevitch et al., 2018) intenta ofrecer un recuento de los orígenes, referentes teóricos y principales tendencias de la investigación en comunicación alternativa en contextos de investigación del Norte del planeta, asumiendo, no obstante, que su carácter sumario obviará inevitablemente muchas referencias importantes. Tampoco pretendemos establecer ningún canon de autores/as o escuelas, sino más bien una aproximación al estado del arte de la investigación europea y anglosajona,

1 Este trabajo se adscribe a las líneas habituales de investigación de la Red de Investigación Comunitaria, Alternativa y Participativa (RICCAP) (www.riccap.org) 
en comparación con la latinoamericana, y con relación a las características de los contextos macrorregionales en los que dichas tradiciones se insertan.

El artículo se divide en tres partes. En primer lugar, se exploran las diferencias entre la investigación latinoamericana y europea. Posteriormente, resumimos las principales matrices de investigación que subyacen en la teoría del Norte prestando especial atención a cinco países europeos (Francia, Reino Unido, Alemania, Italia y España) e incorporando algunas referencias estadounidenses que han legado importantes reflexiones a la materia. Por último, describimos sucintamente algunos trabajos que están contribuyendo a la renovación temática de estas perspectivas y desde los que se vislumbran futuras tendencias de investigación.

\section{Los estudios de comunicación alternativa en Europa y América Latina}

En los tempranos años 20, el sociólogo estadounidense Robert Ezra Park (1922) y el dramaturgo alemán Bertold Brecht (1927) advirtieron respectivamente sobre el potencial emancipatorio de la prensa migrante y de la radio. No obstante, y a pesar de su antigüedad, los estudios sobre comunicación alternativa y comunitaria en Europa y EE.UU. han tenido históricamente un desarrollo bastante disperso y desconectado entre sí. Si bien hay un ligero repunte en las últimas décadas del siglo XX (ej. Armstrong, 1980; Glessing, 1970; Downing, 1984; Mattelart \& Siegelaub, 1979), ${ }^{2}$ no es hasta comienzos de siglo XXI cuando estos trabajos comienzan a cimentar un campo autónomo y diferenciado de investigación, en especial si lo comparamos con áreas afines — como la comunicación para el desarrollo o la economía política de la comunicación — que han sido matrices y con las que el área mantiene continuidades. De hecho, podemos relacionar la institucionalización del campo con el interés suscitado por las potencialidades participativas de la Web 2.0 (Jankowski, 2006), ${ }^{3}$ las innovadoras apropiaciones tecnológicas del movimiento antiglobalización —entre las que destacó Indymedia (Kidd, 2003) - , y la propia conformación de un campo de estudios sobre comunicación y movimientos sociales (Askanius \& Gustafsson, 2010), tras años en los que las teorías de la acción colectiva habían prestado una "atención tangencial a las dinámicas de los medios" (Downing, 2008, p. 41).

2 El listado, por supuesto, es mucho más extenso, en los 70: Berrigan (1977), Díaz Bordenave (1977); los 80: Downing (1984), Lewis et al. (1984), Lewis y Booth (1989), Soley y Nichols (1986); los 90: Girard (1992), Lewis (1993), Jankowski, Prehn y Stappers (1992); y los 2000: Gumucio (2001), Coyer, Dowmunt y Fountain (2007). Aunque es preciso destacar que el tipo de publicación más importante fueron los balances de experiencias en comunicación alternativa y para el desarrollo, si bien estableciendo escasos diálogos entre sí. Uno de los más importantes volúmenes de la época, recientemente traducido al español en un trabajo coordinado por Francisco Sierra, es "Comunicación y Lucha de Clases", de Mattelart y Siegelaub (1979), que sintetiza el pensamiento marxista sobre comunicación alternativa y estructura de la comunicación de la época.

3 Jankowski (2006) compara este tercer momento de investigación con una "primera" y "segunda" olas dedicadas, respectivamente, a la prensa, desde los años 1920, y a los medios electrónicos -radio, vídeo, televisión, etc. - , desde finales de los años 60. 
En comparación con estos contextos, la investigación latinoamericana sobre comunicación alternativa se gestó como campo algo antes, ya a comienzos de los años 70, en un contexto de fuerte resistencia a las políticas verticales y extensionistas de comunicación del desarrollo exportadas desde EE.UU. y las agencias de cooperación de las Naciones Unidas: FAO, PNUD, etc. (Manyozo, 2012). El fracaso de estos planteamientos provocó una fuerte reacción en autores como Luis Ramiro Beltrán o Juan Díaz Bordenave, quienes reivindicaron modelos más horizontales y participativos de comunicación, además de vinculados a las necesidades de expresión y desarrollo de las propias comunidades. Inspirados por la pedagogía dialógica de Freire y las propias experiencias alternativas que surgen a mediados de siglo - como las radios mineras bolivianas o distintos proyectos de educomunicación popular-, los años 70 y 80 fueron el germen de un sinfín de proyectos comunitarios de carácter más reformista o revolucionario y en abierto desafío a las oligarquías locales, los gobiernos autoritarios o las relaciones de dependencia. En estos proyectos, la comunicación y los medios (radio, prensa popular, cartillas, cassettes, teatro, etc.) comenzaron a ser concebidos como instrumentos vitales para la alfabetización, la toma de conciencia crítica, o la mejora de las condiciones de vida de los "oprimidos" y las clases populares (Freire, 1973). ${ }^{4}$ Hasta finales de siglo XX, autores como Máximo Simpson, Fernando Reyes Matta, Rosa María Alfaro, Rafael Roncagliolo, Mario Kaplún, Alfredo Paiva, Marita Mata o Margarita Graziano cimentaron el "alternativismo" (Kaplún, 2019) o una tradición investigativa muy atenta al devenir de las propias experiencias prácticas (Barranquero, 2015, 2020) y conformada desde referencias a la comunicación para el desarrollo, la educación popular, la economía política, la folkcomunicação (en Brasil) y, más adelante, los estudios culturales.

Con relación al contexto mediático, la conformación del campo en Latinoamérica tuvo mucho que ver con la reacción popular frente al dominio de una estructura privada de medios fuertemente concentrada y poco o nada atenta a las necesidades materiales o expresivas de las poblaciones más desfavorecidas. Durante la segunda mitad del siglo XX, el subcontinente no llegó a desarrollar un sistema de medios de servicio público como el que se cimentó en algunos países de Europa y cuyo ejemplo más paradigmático lo constituye la BBC británica. De ahí que los medios alternativos fueron percibidos como una manera de dar "voz" a los sectores populares — por ejemplo, frente a la orientación elitista y blanca de la prensa y la televisión-y de contrarrestar los intereses monopolísticos del sector privado de cara a consolidar estructuras más plurales de comunicación.

4 Nos referimos a un contexto en el que destacaron iniciativas de carácter más integrado y reformista como las emprendidas por comunidades de base, inspiradas por la teología de la liberación y con influencia del Consejo Episcopal Latinoamericano (CELAM), o una concepción más militante como las de los artículos publicados en los Cuadernos del CEREN de Armand Mattelart y su equipo en el contexto de la vía chilena al socialismo. Una completa historia reciente de la comunicación alternativa en Latinoamérica se puede consultar en Badenes (2020). Dos décadas antes, Peppino (1999) hizo lo propio en un estudio circunscrito al ámbito de la radio. 
En Europa, los estudios sobre comunicación alternativa se desarrollaron, en cambio, en un contexto en el que ya existía una tradición de servicio público. Es por ello que las luchas de las primeras radios libres estuvieron, sobre todo, orientadas a conseguir un espacio ciudadano en el espectro radioeléctrico, ${ }^{5}$ más que a cubrir perentorias necesidades de alfabetización o luchar contra la concentración mediática y la dependencia cultural del "Tercer Mundo", que fueron dos de los ejes centrales del Informe McBride (1980). ${ }^{6}$

Otra diferencia apreciable es que la comunicación alternativa en Europa y EE.UU. recibió un fuerte influjo de las teorías cimentadas en torno a manifestaciones de carácter underground y contracultural en las artes plásticas, audiovisuales (cine y vídeo) o el teatro, con lo que la reflexión en clave culturalista, performativa y artística de algunos trabajos se hace más evidente que en América Latina, en especial hasta finales de siglo XX (Baldelli, 1977; Plant, Mueller, 1989). Asimismo, y aunque EE.UU. o Europa no estaban exentos de desigualdades, algunos trabajos de referencia hasta finales de siglo XX tuvieron la marca de búsquedas posmaterialistas (Inglehart, 1977). En otras palabras, la lucha de la comunicación alternativa europea no siempre se relacionó con necesidades materiales como la supervivencia o el trabajo, sino más bien con luchas por la autoexpresión individual, el medioambiente, la paz, o el reconocimiento cultural, que eran reivindicaciones algo menos evidentes en dicha época en América Latina. Las reflexiones en el Norte también recibieron mucha influencia de los movimientos sociales juveniles del período como las revueltas en torno a Mayo del 68 (Lefebvre, 2011), además de marcos teóricos y valores libertarios procedentes de los estudios del lenguaje — semiótica, (post)estructuralismo, entre otros-, el marxismo, el anarquismo o la tradición autonomista y operaria en Italia (Downing, 2011). De hecho, el sujeto al que se dirigen los estudios de finales de siglo en Europa no es tanto el genérico "oprimido" latinoamericano - definido en términos de clase social y muy dependiente de enfoques como las teorías de la dependencia-, sino más bien los grupos y culturas "subalternas" a los que apelaron los Estudios Culturales (y que incluye desde migrantes a mujeres, jóvenes, colectivos LGTBIQ, etc.), además de organizaciones militantes de izquierda y colectivos contraculturales. Ya en las postrimerías del siglo XX, se populariza tanto en América Latina como en el Norte una apelación más genérica a la noción de "ciudadanía” (Rodríguez, 2001), que, aunque también contestada, pone el foco en las apropiaciones

5 O incluso lucharon por la necesidad de emitir por emitir, más allá de cualquier reivindicación política, social o educativa, como afirma Pérez Martínez (2018).

6 Dicho Informe fue muy influyente en la conformación de la tradición investigativa latinoamericana por cuanto reconocía el derecho a la comunicación de las comunidades y la necesidad de dotarlas de estructuras autónomas de medios. No obstante, también se señala que el propio desencanto al comprobar que las premisas del McBride no estaban surtiendo efecto en Latinoamérica, pudo estimular la investigación en comunicación alternativa durante las décadas de los 80 y los 90 en que se publican compendios tan difundidos como los de Simpson (1986) o Reyes Matta (1983). 
comunitarias de los medios para el ejercicio político de la ciudadanía y que, de alguna manera, puede ayudar a abrir un diálogo entre ambas tradiciones.

Como señalábamos, en los estudios anglosajones hay que esperar hasta la década de los 90 para avistar cierta institucionalización del campo. En este sentido, conviene destacar dos momentos clave: el primero, que coincide con la publicación, en torno a 2001, de tres trabajos ampliamente difundidos en el contexto anglosajón y que ayudaron a renovar las reflexiones en el ámbito (Downing, 2001; Rodríguez, 2001; Atton, 2001), y el segundo, que apunta a la multiplicación de las formas expresivas alternativas a partir de las nuevas posibilidades brindadas por el entorno digital. En específico, fueron muy importantes las reflexiones generadas a partir de las revueltas zapatistas en Chiapas desde 1994 y el movimiento anti/alterglobalización desde finales de los años 9o. Dichos hitos marcaron el inicio de una conciencia global acerca del poder de las tecnologías digitales para la difusión y conexión de las protestas, que, a veces, incluso recayó en un exceso de tecnodeterminismo (Rodríguez, Ferron, \& Shamas, 2014). Dicho interés acabó por cimentar una línea importante de reflexiones y trabajos empíricos sobre activismo, movimientos y comunicación (en la que algunos/as incluyen a los medios alternativos), que tardó algo más en llegar a América Latina. Allí, los estudios sobre acción colectiva se popularizaron en las primeras décadas del siglo XXI y entraron pronto en diálogo con nociones como las de desarrollo y cambio social y, más recientemente, con el debate sobre la decolonialidad (Kaplún, 2019).

Como estamos sugiriendo, la cuestión de las denominaciones y los énfasis también varían en el contexto latinoamericano y anglosajón. Trabajos como los de Ferron (2012) advirtieron de la existencia de hasta 50 denominaciones para nombrar un campo que es más complejo y diverso que el de los medios convencionalesyen elquelasetiquetas académicasconvivencon denominaciones derivadas de las propias prácticas. Todos los apellidos son "situados" y dependen mucho de su contexto de origen. De hecho, las etiquetas "popular" y "desarrollo" siguen teniendo una fuerte raigambre en Latinoamérica, mientras que en el ámbito anglosajón han tenido una vital influencia las "nuevas" denominaciones de medios radicales (Downing, 1984/200o) o ciudadanos (Rodríguez, 2001). La etiqueta "alternativa" se trabaja en ambos contextos mientras que la "comunitaria" se ha popularizado en todo el planeta, en buena medida porque es el concepto que más se maneja en el ámbito de la regulación audiovisual e incluso en las grandes organizaciones profesionales -AMARC, Community Media Forum Europe (CMFE) — y académicas del sector. ${ }^{7}$ Por último, en América Latina son populares las etiquetas vinculadas a la tradición de la comunicación para el desarrollo (horizontal, participativa, para la liberación y, recientemente,

7 Aquí nos referimos, por ejemplo, a la Association for Media and Communication Research (IAMCR), que desde principios de siglo XXI tiene una sección dedicada a Community Communication (ComCom), que en 2016 añadió el complemento de "Alternative Media", hasta conformarse como la Sección Community Communication and Alternative Media (http://iamcr.org/s-wg/section/community-communication). 
decolonial) y tanto en América Latina como en EE.UU. o Europa hay reflexiones que vinculan lo alternativo con la democracia, los derechos y, en específico, el derecho a la comunicación (d'Arcy, 1969).

Por último, conviene señalar que en el contexto francés son importantes las discusiones en torno a la denominación de "medios asociativos", además de "libres", que es una etiqueta bastante común en Francia, España o Alemania. En Italia, se han explorado conceptos como el de contrainformación (Baldelli, 1977), mediactivismo (Berardi Bifo, 2006; Pasquinelli, 2002) o televisión de calle (Ardizzoni, 2009). Finalmente, España suele actuar como "bisagra" de ambas tradiciones por su cercanía cultural y lingüística con Latinoamérica y su adscripción europea. Desde los inicios de la transición a la democracia y la conformación de las primeras facultades independientes de ciencias de la información, se organizaron encuentros (ej. Vidal Beneyto, 1979) que congregaron perspectivas de ambas regiones, por lo que España importó tanto las reflexiones latinoamericanas de la educomunicación o la comunicación para el desarrollo como aportes europeos relacionados con la radios libres, el postestructuralismo o la contrainformación y el détournement situacionista. Ya en tiempos residentes, han comenzado a cimentar teorizaciones propias como las del "ciberactivismo" y las "tecnopolíticas", más adscritas a los estudios de comunicación y movimientos sociales, o la del "tercer sector de la comunicación”, más arraigadas a la reflexión propia de la comunicación alternativa.

La Tabla 1 muestra las principales diferencias detectadas entre el campo latinoamericano de la comunicación alternativa y popular y los estudios sobre todo, anglosajones.

Tabla 1. Diferencias entre las tradiciones de comunicación alternativa en Latinoamérica y Europa

\begin{tabular}{|l|l|l|}
\hline & Latinoamérica & Europa (y EE.UU.) \\
\hline Origen del campo & $\begin{array}{l}\text { Reflexiones precursoras desde principios } \\
\text { de los 70 (Freire, Beltrán, Kaplún, Díaz } \\
\text { Bordenave, etc.). }\end{array}$ & $\begin{array}{l}\text { Precedentes en los años 20 (Park, Brecht) } \\
\text { y repunte desde los 80 (Downing, Lewis, } \\
\text { etc.). }\end{array}$ \\
\hline Institucionalización & $\begin{array}{l}\text { Desde los 80 (Simpson, 1986; Reyes Matta, } \\
\text { 1983). }\end{array}$ & $\begin{array}{l}\text { Desde 2001 en adelante (Downing, 2001; } \\
\text { Atton, 2001; Rodríguez, 2001). }\end{array}$ \\
\hline $\begin{array}{l}\text { Experiencias } \\
\text { fundacionales }\end{array}$ & $\begin{array}{l}\text { Radios sindicales mineras, radiofórums y } \\
\text { radioescuelas (Radio Sutatenza / ACPO) } \\
\text { (desde los 50); proyectos de alfabetización } \\
\text { con apoyo de medios (método Freire, } \\
\text { cassette-foro y telenovelas de Kaplún); tercer } \\
\text { ciney vídeo/cine participativo y comunitario; } \\
\text { teatro del oprimido (desde los 60), etc. }\end{array}$ & $\begin{array}{l}\text { Experiencias radiofónicas o } \\
\text { cinematográficas de las vanguardias } \\
\text { (años 20), movimientos artísticos } \\
\text { contraculturales de los 60 (CoBrA, } \\
\text { Internacional Situacionista), radios libres } \\
\text { en Francia, Italia y Reino Unido y acciones } \\
\text { culturales alternativas Mayo del 68, low } \\
\text { power broadcasting en EE.UU., etc. }\end{array}$ \\
\hline $\begin{array}{l}\text { Contexto histórico } \\
\text { siglo XX }\end{array}$ & $\begin{array}{l}\text { Relaciones de dependencia y luchas por } \\
\text { la liberación; movimientos reformistas y } \\
\text { revolucionarios desde mitad de siglo XX: } \\
\text { Revolución Cubana, Vía chilena al socialismo, } \\
\text { etc.; dictaduras en el Cono Sur a finales de } \\
\text { siglo, etc. }\end{array}$ & $\begin{array}{l}\text { Reconstrucción de Europa durante la } \\
\text { posguerra, progresivo Estado de Bienestar } \\
\text { ysatisfacción de valores "materiales"; } \\
\text { movimientos de protesta (ej. Mayo del 68) } \\
\text { posmateriales: pacifismo, ambientalismo, } \\
\text { luchas por derechos civiles en EE.UU., etc. }\end{array}$ \\
\hline
\end{tabular}




\begin{tabular}{|l|l|l|}
\hline $\begin{array}{l}\text { Contexto mediático } \\
\text { siglo XX }\end{array}$ & $\begin{array}{l}\text { Monopolios privados y fuerte dependencia } \\
\text { externa de contenidos e ideología } \\
\text { (Latinoamérica como "patio trasero" de } \\
\text { EE.UU.); ausencia o extrema debilidad de } \\
\text { medios públicos. }\end{array}$ & $\begin{array}{l}\text { Consolidación de un sistema público de } \\
\text { medios y tendencia a la privatización (o } \\
\text { privatización extrema) de la industria } \\
\text { mediática y el espacio radioeléctrico en } \\
\text { Europa. }\end{array}$ \\
\hline $\begin{array}{l}\text { Enfoques de } \\
\text { las primeras } \\
\text { experienciasy } \\
\text { teorías }\end{array}$ & $\begin{array}{l}\text { Educary empoderar a los grupos "oprimidos"; } \\
\text { fortalecer a la comunidad frente a la ausencia } \\
\text { de lo público; enfrentar oligarquías y } \\
\text { dictaduras, etc. }\end{array}$ & $\begin{array}{l}\text { Representar grupos y culturas } \\
\text { "subalternas"; generar un tercer espacio } \\
\text { mediático al margen del servicio público y } \\
\text { los medios comerciales, etc. }\end{array}$ \\
\hline $\begin{array}{l}\text { Matrices } \\
\text { epistemológicas en } \\
\text { los años 70 }\end{array}$ & $\begin{array}{l}\text { Comunicación para el desarrollo, economía } \\
\text { política, teoría de la dependencia, educación } \\
\text { popular, folk-comunicación, etc. }\end{array}$ & $\begin{array}{l}\text { Teorías críticas y estudios culturales, } \\
\text { posestructuralismo, (pos)marxismo, } \\
\text { anarquismo, perspectivas artísticas } \\
\text { contraculturales... Ya en el siglo XXI, } \\
\text { movimientos sociales. }\end{array}$ \\
\hline $\begin{array}{l}\text { Denominaciones } \\
\text { más populares }\end{array}$ & $\begin{array}{l}\text { Comunicación alternativa, popular, para el } \\
\text { desarrollo y el cambio social, decolonial, } \\
\text { horizontal, participativa, ciudadana, etc. }\end{array}$ & $\begin{array}{l}\text { Comunicación alternativa, ciudadana, } \\
\text { radical, contrainformación, mediactivismo, } \\
\text { medios libres, etc. }\end{array}$ \\
\hline
\end{tabular}

Fuente: Elaboración propia

\section{Las matrices comunicacionales de la reflexión europea}

La investigación en comunicación alternativa y comunitaria emerge en Europa a partir de distintas escuelas y tradiciones epistemológicas. A continuación, planteamos un recorrido sumario fijándonos en las primeras y principales inspiraciones que recibe el campo de la comunicación alternativa en distintos países europeos.Enprimerlugar,y coetáneay conocidadeBrecht(1927),laEscuela de Frankfurt legó una importante crítica a la estandarización de la cultura desde conceptos como los de "industria cultural" de Adorno y Horkheimer. Además de criticar la pérdida de "aura” en la producción mecanicista de la cultura, Walter Benjamin (1934) reivindicó una superación de los roles tradicionales entre autor y lector con el fin de subvertir la cultura dominante. Ya a mediados de los 60, se cimenta otra matriz de los estudios de comunicación alternativa: los Estudios Culturales británicos que emergen a mediados de los 60. Estos contribuyeron a legitimar problemáticas hasta entonces desatendidas por la investigación comunicacional tales como la cultura popular (que ya comenzaba a conformar una tradición autónoma de trabajos en América Latina por aquellos años) y las resignificaciones de la cultura masiva por parte de culturas subalternas y populares. Sin olvidar las importantes reflexiones de Thompson, Hoggart o Hall, en los medios alternativos siguen siendo inspiradoras las teorías de la comunicación democrática de Raymond Williams (ver Thomas, 2017) y las relecturas de la obra de Antonio Gramsci para comprender la capacidad de agencia de las culturas populares frente a los elementos reproductores de la cultura masiva. ${ }^{8}$

8 Los trabajos de Gramsci proyectaron una enorme influencia también en Latinoamérica, donde ayudaron a revitalizar y complejizar los estudios de comunicación alternativa, en especial a partir de la publicación de De los medios a las mediaciones de Jesús Martín-Barbero (1987). Dicho trabajo rompió con las dicotomías propias de la comunicación alternativa de los años 70 y 80 -lo masivo frente a lo popular, lo vertical frente 
Desligada de los Estudios Culturales, la teoría crítica francesa alcanzó su cénit alrededor de los acontecimientos revolucionarios de Mayo del 68. Décadas antes, las vanguardias artísticas habían legado algunas reflexiones importantes sobre el poder subversivo del arte, cuyo testigo sería retomado en los 60 por movimientos como la Internacional Situacionista, en la que sobresalen los desarrollos de Guy Debord (1967) sobre la espectacularización de la cultura de masas. Paralelamente, una tradición amplia de estudios del lenguaje y sus sentidos (Barthes, Derrida, etc.) ayudaron a reivindicar la autonomía de los sujetos y los significados más allá de las estructuras dominantes. Por su parte, el francés Michel de Certeau (1980) propuso una popular distinción entre las "estrategias" de poder de las industrias culturales y las "tácticas" o respuestas contraculturales de los sectores subordinados.

En Alemania, en 1962 se publica un texto decisivo para la reflexión sobre el potencial de los medios en la conformación de la esfera pública. En su Historia y crítica de la opinión pública, el filósofo Jürgen Habermas (1962), de la segunda generación de la Escuela de Frankfurt, reivindicó el nacimiento de la esfera pública burguesa como manifestación alternativa al absolutismo y elemento configurador de la Modernidad. Años más tarde, Habermas sería contestado tanto por teóricas feministas como Nancy Fraser como por autores frankfurtianos como Alexander Kluge y Oskar Negt. Respectivamente, estos criticaron el olvido en sus teorías del sujeto femenino y de las propias esferas públicas proletarias, una última tesis que está en sintonía con la reivindicación de las manifestaciones populares incluso durante la Edad Media del ruso Bakthin (1965). 9 Desde un marco distinto, el intelectual Hans Magnus Enzensberger (1970) también reflexionó en sus escritos acerca del potencial bidireccional y "emancipatorio" de los medios audiovisuales frente a los usos "represivos" de la cultura de masas.

En la década de los 70 se sitúan los primeros análisis sobre un fenómeno que alcanzaría su cénit en las postrimerías de Mayo del 68 en países como Italia o Francia: las denominadas radios libres o piratas. Este fenómeno fue crucial porque inició una reflexión muy prolífica acerca de la necesidad de liberar el espacio radioeléctrico para incorporar un tercer sector de la comunicación, en un escenario dominado por medios públicos y, sobre todo, medios comerciales concentrados en grandes monopolios. De esta época también datan los trabajos del psicoanalista Félix Guattari, quien, tras participar en experiencias pioneras como radio Alice en Italia —y posteriormente radio Verte en Francia—, demostró

a lo horizontal, lo grande frente a lo pequeño, etc.- y ayudó a vislumbrar los profundos vínculos existentes entre lo masivo, lo mediático y lo popular. Esta complejidad es ya evidente en la obra de autoras de la comunicación ciudadana como Clemencia Rodríguez (Barranquero, 2015, p. 21). Algunas de las reflexiones contenidas en los apartados 3 y 4 fueron planteadas en Barranquero y Treré (2020), si bien han sido retomadas, actualizadas y mejoradas en este ensayo.

9 Las propias críticas a la concepción burguesa y patriarcal de la esfera pública "habermasiana" exceden los propósitos del estudio, pero provocaron que el propio Habermas replantease sus tesis iniciales para incluir esferas al margen de la burguesa con base en las reediciones realizadas desde 1990. 
cómo los medios libres son un espacio de conflicto alrededor de la producción de subjetividad (Guattari, 1978). También en Italia, los textos de Deleuze y Guattari o las experiencias situacionistas inspiraron las reflexiones de Franco 'Berardi' Bifo (2006), quien, tras el cierre forzado de Radio Alice, se refugió en Francia con Guattari y emprendió una extensa producción ensayística que también destacó el potencial subversivo de las radios libres o la "televisión de calle" (Orfeo TV / Telestreet), una experiencia emprendida junto con otro fundador de la citada emisora: Giancarlo Vitali Ámbrogio (Berardi, Jaquemet, \& Vitali, 2004). El panorama italiano se completa con el influyente aporte de Umberto Eco (1974) sobre la posibilidad de alterar los signos dominantes, o "guerrilla semiótica", que ha inspirado otra línea de trabajo más importante en Europa que en Latinoamérica: los estudios de contrapropaganda, sabotaje informativo, culture jamming y subverting. Bien representada por experiencias como las de Luther Blissett en Italia o Consume hasta morir en España, esta perspectiva no está tan relacionada con la creación o apropiación de medios, sino con estrategias basadas en la recodificación y subversión de los productos culturales o publicitarios de la cultura de masas.

Junto a estos planteamientos, en los últimos años los estudios sobre movimientos sociales han comenzado a percatarse de la importancia de los medios y las tecnologías informacionales, ya sea como repertorios de acción colectiva, o como espacio de construcción de identidades y objetivos políticos. En los años 70 y 80, trabajos pioneros como los de los británicos Halloran, Murdoch y Elliott (1970) o Todd Gitlin (1980) en EE.UU. pusieron el foco en la relación entre medios y protestas sociales, a los que siguieron las importantes tesis de Alberto Melucci (1996), quien consideraba que los movimientos sociales son medios en sí mismos al intentar subvertir los códigos imperantes. Trabajos posteriores como los de Dieter Rucht, Donatella della Porta, Manuel Castells, Paolo Gerbaudo o Alice Mattoni han enfatizado en el potencial de estas prácticas mediáticas para configurar la identidad, la movilización de recursos, o la interacción con grupos externos. Otra tradición en el ámbito es el propio aporte de los estudios en comunicación para el desarrollo y para el cambio social, que tradicionalmente ha estado centrada en los países del Sur del planeta y que en España se ha entendido a veces como la comunicación del ámbito de las ONG y las agencias de cooperación. En esta línea, los escritos del belga Jan Servaes (1999), la argentina afincada en Europa Florencia Enghel (Enghel \& Noske-Turner, 2018) o del danés Thomas Tufte (Tufte \& Mefalopulos, 2009) son referencia. Este último fue coeditor, junto con Alfonso Gumucio, de la primera antología de lecturas clásicas y contemporáneas en la subdisciplina (Gumucio \& Tufte, 2006).

Recopilando la tradición de la comunicación alternativa de distintos contextos, en 1984, el estadounidense John Downing publicó la primera versión de su Radical Media, que habría de retomar y actualizar en 2001, marcando uno de los hitos para la institucionalización del campo en el ámbito europeo 
y anglosajón, también con cierta presencia en Latinoamérica. Su ambicioso libro describió las importantes influencias que recibe esta tradición desde el anarquismo, la nueva izquierda y la tradición autonomista italiana y exploró experiencias tan varias como las radios libres italianas, los fundamentos críticos del culture jamming o el samidzat en la antigua Unión Soviética (Downing, 1984, 2001). A finales de los 2000, editó el que es tal vez el intento más ambicioso realizado hasta la fecha de sistematizar la relación entre movimientos sociales y comunicación prestando especial atención a los medios libres y comunitarios: Encyclopedia of Social Movement Media (Downing, 2010), al que seguiría otro importante volumen internacional coordinado por Chris Atton (2015).

\section{Referentes y debates contemporáneos en el contexto europeo}

Una vez revisadas obras pioneras, las siguientes líneas presentan el trabajo de unas corrientes y autores que consideramos representativos de las temáticas que hoy dominan la investigación. No obstante, reiteramos que este balance no pretende atrapar toda la riqueza de unos estudios que hoy se encuentran en proceso de expansión, sino que es más bien una invitación a la lectura de pensadores y pensadoras que están ayudando a complejizar la perspectiva. Desde la corriente de los Cultural Studies, el británico Chris Atton es uno de los teóricos más relevantes de la investigación sobre medios alternativos en Europa. Inspirado por el estadounidense John Downing, el autor ha aportado una de las definiciones más afinadas y completas de "medios alternativos", a los que contempla, por un lado, en relación con el contexto sociocultural entendiéndolos como "prácticas"- y, por otro, de acuerdo a su carácter en tanto "textos" y contenidos (Atton, 2001, 2004). Para el autor, la simple cuestión del contenido no es suficiente para definir este complejo universo, puesto que los medios alternativos son productores de cambio social, en especial por activar unas dinámicas de organización — horizontales y asamblearias- que amplían la participación ciudadana en comparación con las lógicas unidireccionales de los masivos. El académico distingue además entre "productos" y "procesos". Entre los primeros, incluye el contenido, las formas y las adaptaciones e innovaciones temáticas de los medios alternativos. En los procesos, abarca cuestiones como su uso distributivo, los diferentes roles y responsabilidades que en ellos se dan, y, por último, la mutación de los propios procesos de comunicación en virtud de la creación de redes horizontales que transforman las relaciones sociales.

Inspirado por los Estudios Culturales, pero también en diálogo con otras perspectivas, el británico Nick Couldry argumenta que la tarea más importante de los medios alternativos es desafiar el sistema altamente concentrado de los medios masivos y, sobre todo, retar su poder simbólico desde la superación de su "atrincherada división del trabajo (productores de historias contra consumidores de historias)" (Couldry, 2003, p. 45). Su potencial emancipador reside para el autor en su capacidad de abrir el acceso a la producción mediática 
a un público amplio y plural, lo que proporciona nuevas versiones de la realidad frente al relato estereotipado y las formas de "nombrar" el mundo que caracterizan a los medios de corte neoliberal. El autor realiza asimismo dos aportes fundamentales para comprender su función social. En primer lugar, el académico responde a la crisis financiera de 2008 a partir de sus reflexiones en torno al concepto de "voz" (voice), al que percibe como un verdadero agente de cambio, puesto que es un proceso que incluye la capacidad de "dar cuenta de sí mismo y de lo que afecta a la vida de uno” (Couldry, 2010, p. 3). Couldry entiende la voz como "proceso" —o la capacidad que tienen algunos grupos sociales de hablar y de encontrar medios de expresión propios-, pero asimismo como "valor", que implica la cualidad de ser apreciado, reconocido y tenido en cuenta en una perspectiva desde la que reivindica las historias procedentes de los ciudadanos como seres narrativos (Couldry, 2010, pp. 7, 13). En segundo lugar, los analiza como "prácticas mediáticas" (media practices) (Couldry, 2004; 2012), invitando a superar los enfoques funcionalistas que conciben a estos medios como simples herramientas o textos. Couldry desafía los enfoques instrumentales dominantes sobre la base de un concepto propio de "mediaciones" tecnológicas, que se inspira en la propia noción de Jesús Martín-Barbero (1987) y que contribuye a tejer puentes entre la comunicación alternativa y la literatura sobre movimientos sociales y cultura digital.

Esta perspectiva ha impactado de forma significativa en una nueva generación de investigadores de medios digitales y movimientos sociales (Barassi, 2015; Cammaerts, Mattoni \& McCurdy, 2010; García García \& Treré, 2014; Stephansen \& Treré, 2019; Uldam \& Askanius, 2013; Treré, 2012, 2019), que, desde su enfoque, han empezado a explorar lo que la gente "hace" cuando se apropia de las tecnologías, así como el conjunto de creencias que guían la actuación de los propios activistas mediáticos, también en la línea que advertía Clemencia Rodríguez (2001) en su trabajo sobre los "medios ciudadanos". Por ejemplo, la noruega Hilde Stephansen ha logrado combinar su teorización sobre las "prácticas" con las reflexiones sobre "medios ciudadanos" (Stephansen, 2013, 2016; Mahony \& Stephansen, 2016; Stephansen \& Treré, 2019). Su trabajo intenta superar la fijación histórica de muchos académicos con el mensaje para explorar la amplia gama de experiencias socialmente situadas y proponer un cambio radical de enfoque: desde los "medios ciudadanos" a las "prácticas alrededor de los medios ciudadanos" (Stephansen, 2016). Esta nueva perspectiva le permite estudiar tres dimensiones distintas: por un lado, un espectro más amplio de prácticas alternativas, más allá de las directamente relacionadas con el contenido de los medios; por otro, las diferentes formas de agencia que estas instauran; y, por último, el tejido social que las mismas contribuyen a generar mediante su interrelación con los movimientos sociales y la ciudadanía organizada. La deuda de este enfoque con la tradición latinoamericana y la necesidad de un diálogo más sistemático están explícitamente articulados en una reciente colección de contribuciones que recoge autores europeos y 
latinoamericanos reflexionando sobre las conexiones entre prácticas mediáticas y medios ciudadanos (Stephansen \& Treré, 2019; sobre temáticas afines ver Pertierra \& Salazar, 2019).

Con el fin de reforzar la idea de que los medios alternativos juegan un papel de red dentro de la sociedad civil, el profesor belga Nico Carpentier sugiere el uso de la metáfora del "rizoma" de Deleuze y Guattari (1987) como una nueva tipología. De hecho, la naturaleza no lineal, anárquica y nómada del rizoma es utilizada para simbolizar el papel que estos tienen a la hora de promocionar redes fluidas de organizaciones y personas. Este papel se combina, además, con su capacidad para desterritorializar a su contraparte: los medios tradicionales, a los que define desde un "modelo arbólico" que representa a la filosofía tradicional del Estado y de los poderes convencionales. El enfoque rizomático comprende los medios alternativos como nodos esenciales para la organización en red de la sociedad civil, unos nodos que ayudan tanto a mantener los vínculos sociales como a crear nuevas interacciones entre estas entidades, los movimientos sociales y la propia ciudadanía. Por último, la metáfora le permite enfatizar en las numerosas interacciones que los medios alternativos entretejen con los actores estatales y del mercado, ya que estas entidades no operan en el vacío, sino que crean vínculos problemáticos con los anteriores, intentando no perder su propia identidad (Carpentier, Lie, \& Servaes, 2003; Bailey, Carpentier \& Cammaerts, 2008; Carpentier, 2015; Santana \& Carpentier, 2010).${ }^{10}$ El autor ha insertado sus reflexiones sobre medios alternativos dentro del marco conceptual más amplio de la "participación”, investigando el uso de esta noción escurridiza que ha tendido a ser cooptada por parte de políticos y corporaciones mediáticas hasta vaciarla de su significado político y emancipador. Carpentier subraya que la participación es muchas veces confundida con la visión acrítica de un periodismo ciudadano que es celebrado y publicitado por los medios convencionales como participación cuando en realidad no lo es (Carpentier, 2011).

Por su parte, el austríaco Christian Fuchs, en colaboración con Marisol Sandoval, argumenta que los medios alternativos deben ser considerados desde la óptica o concepto de "medios críticos". Partiendo de la crítica a la confusión conceptual que se ha dado en el campo en torno a la idea de lo alternativo, Sandoval y Fuchs consideran que estos medios son herramientas que deben proteger la dimensión "humana" del ser, abogando por un humanismo radical y oponiéndose a todo tipo de dominación (Fuchs, 2010; Sandoval \& Fuchs, 2009). Los autores argumentan que se requiere una gran cantidad de recursos con el fin de obtener visibilidad en la esfera pública dentro de un sistema capitalista. Esto

10 Así, por ejemplo, en su análisis de las radios belgas Paniky Aire Libre, Santana y Carpentier (2010) demuestran que, además de los muchos vínculos con las organizaciones de la sociedad civil, estas emisoras suelen interactuar con la esfera del Estado - desde la petición de ayudas y programas-y a partir de aquí se inicia un problemático proceso para el sostenimiento de la autonomía y la misión social frente a las posibles presiones políticas o económicas. 
no significa que los proyectos radicales de pequeña escala no sean importantes, sino que estos deberían de abandonar una idea ingenua de autonomía a toda costa y recurrir, más bien, a las técnicas de producción y difusión mediáticas propias del sistema neoliberal, que a veces pueden ser útiles con el fin de alcanzar objetivos progresistas dentro de un marco capitalista omnipresente que deja pocos resquicios para lo contrahegemónico. Para ellos, el requisito mínimo para hablar de medios alternativos vuelve a residir en la presencia de contenido crítico definido, en la línea de la tradición marxista, como aquello que desafía las formas tradicionales de opresión y dominación, y que promueve una visión de sociedad humanizada, razonable y autodeterminada que es posible lograr mediante las luchas sociales y de clase.

En otro orden de cosas, en 1997, un grupo de activistas y teóricos de los medios de origen europeo y estadounidense - entre los que destaca el holandés Geert Lovink, y otros autores como David García y Joanne Richardsonpublicaron un libro ampliamente divulgado entre los circuitos artísticos y mediáticos alternativos: el $A B C$ de los tactical media. ${ }^{11}$ Desde una perspectiva más tecnológica, los autores argumentan que los medios tácticos son el producto de "una revolución en el ámbito de la electrónica doméstica y de las formas expandidas de distribución (desde acceso público al cable hasta Internet) y su consiguiente explotación por individuos o grupos que se sienten oprimidos o excluidos de una cultura expandida". Los "tactical media" privilegian las intervenciones rápidas y rechazan las creaciones permanentes, duraderas e "ideológicas" de los medios convencionales. Esto lleva a los autores a criticar las dicotomías clásicas que se han dado en este ámbito entre lo alternativo y lo popular, lo privado y lo público y lo amateur y lo profesional, y se remiten más bien a trabajos como el de Michel de Certeau (1984) para acuñar un nuevo vocabulario de "tácticas" e intervenciones artísticas y activistas. Algunos creadores han aplicado esta concepción también para describir el papel de los medios en las insurrecciones surgidas desde 2011 (Kluitenberg, 2011).

Desde mediados de los 200o, conviene prestar atención al trabajo realizado en tiempos recientes por la investigadora italiana Stefania Milan. En su libro Social Movements and their Technologies, Milan (2013) explora las interacciones entre los movimientos de protesta y sus tecnologías liberadoras y "liberadas", centrándose en la irrupción de proyectos radicales en el ámbito de Internet. La investigadora analiza de qué manera grupos tecnológicos como los hacktivistas han ido creando con el paso de los años fórmulas alternativas autónomas y clandestinas con respecto a los sistemas de comunicación ordinarios, hasta conseguir moldear e impactar en las formas de interacción de muchos colectivos. De esta manera, Milan inserta el activismo mediático dentro de la literatura sociológica sobre los movimientos sociales y la acción colectiva, acortando la brecha entre ambos campos. La autora posiciona a las organizaciones, activistas

11 Verhttp://aleph-arts.org/pens/abc.html 
y colectivos alternativos en el marco más amplio del movimiento transnacional por el derecho a la comunicación, desde el que invita a estudiar la perspectiva de las políticas mediáticas y de las luchas alrededor de los marcos reguladores de la red y las plataformas digitales. En los últimos años, la investigadora también ha explorado las prácticas relacionadas con lo que denomina el "activismo de datos" (data activism), que emerge cuando la ciudadanía adopta una postura crítica hacia los big data, que se apropia y manipula para hacer campaña y promover el cambio social” (Milan y Gutiérrez, 2015). En el contexto de una creciente y ubicua "dataficación" de las prácticas e interacciones diarias, Milan explora cómo algunos ciudadanos y organizaciones se apropian de las tecnologías de datos para reaccionar frente a la vigilancia omnipresente y la violación de derechos civiles causada por la intrusión gubernamental y corporativa (a la que denomina "activismo de datos reactivo"). De esta manera, la sociedad civil está avanzando en la construcción de un "activismo de datos proactivo" que sitúa los mismos al servicio del compromiso cívico y el cambio social y que, de alguna manera, conecta de nuevo con las reflexiones sobre la noción de "medios ciudadanos" de Clemencia Rodríguez (2001). Según Milan, el activismo de datos representa una nueva forma de medios ciudadanos puesto que coloca en su centro una aproximación crítica hacia los big data y, sobre todo, porque desafía la concepción institucional que restringe la ciudadanía al momento del ejercicio del voto mediante apropiaciones tecnológicas que amplían los espacios políticos más allá de los lugares institucionales. Finalmente, la ecología de medios es una prometedora perspectiva conceptual que ha surgido en los últimos años para superar determinados reduccionismos que se observan en el estudio de las relaciones entre movimientos sociales, tecnologías de la información y prácticas alternativas de comunicación (Treré, 2020). Este abordaje utiliza la metáfora y los marcos de la "ecología de los medios" para explorar la riqueza y entender la complejidad de las formaciones alternativas contemporáneas (Mercea, Iannelli, \& Loader, 2015; Treré \& Mattoni, 2016). Así, Treré destaca que una visión ecológica puede superar las falacias del reduccionismo comunicativo que caracteriza la literatura sobre acción colectiva, puesto que "permite ver los movimientos sociales como configuraciones complejas múltiples prácticas, actores e infraestructuras que están interconectadas" (Treré, 2020, p. 34). Esta visión permite superar antiguas dicotomías como online/offline, viejo/nuevo, global/ local, interno/externo y organizacional/cultural, reconociendo la complejidad, la multiplicidad y la hibridez entre formas y prácticas comunicativas activistas y la riqueza de los repertorios de acción de los movimientos sociales. Este enfoque también invita a realizar análisis diacrónicos de las prácticas relacionadas con los medios activistas para superar la miopía cortoplacista de muchos estudios actuales, cuyo enfoque se centra únicamente en las últimas -y más seductoras - apropiaciones tecnológicas. Finalmente, esta visión destaca la importancia de reconocer la naturaleza política y crítica de las media ecologies, reconociendo las limitaciones y los riesgos de las incursiones corporativas y 
mainstream, y afinando las herramientas para alcanzar un verdadero cambio social a través de la comunicación. ${ }^{12}$

\section{Conclusiones}

A grandes rasgos, la tradición investigadora en comunicación alternativa y comunitaria en Europa es dilatada en el tiempo y se nutre defuentes y trayectorias teórico-metodológicas diversas, tal y como ocurre en Latinoamérica. De hecho, las líneas que componen este ensayo comprueban que resulta extremadamente complejo arribar a una propuesta unificadora, en especial porque el universo de las prácticas alternativas es multiforme, varía en cada contexto y distintos estudios han venido advirtiendo sobre la imposibilidad de crear una teoría unificadora y universal, más allá de las concomitancias que guardan entre sí las diferentes experiencias. Por otro lado, y más allá de que existen algunas características comunes a todo el territorio europeo - como la progresiva integración del territorio en la Unión Europea-, las diferencias culturales y lingüísticas han dado lugar a cierto aislamiento de las comunidades académicas, que se han acabado configurando en torno a ciertos referentes locales e incluso han tendido a establecer entre sí menos puentes que la tradición crítica latinoamericana, que durante y hasta finales de siglo XX mantuvo ciertas constantes temáticas como la preocupación acerca del eje dependencia y praxis para la liberación.

En Europa nos encontramos, en suma, frente a una tradición de estudios en exceso localizada que, en sus versiones más extremas, llega a desconocer la extensa historia acumulada por los profesionales y activistas del campo en otras latitudes, tal y como denunciaban hace unos años Rodríguez, Ferron y Shamas (2014). Buen ejemplo de esto es una larga pléyade de trabajos recientes que, en su tecno-fascinación, se han aproximado a tecnologías como la Web 2.0, desde una acusada falta de perspectiva histórica y desconociendo, por lo demás, la larga tradición de estudios acerca de la comunicación alternativa latinoamericana y su reivindicación de la participación como dimensión central de lo alternativo. Otra marca común de la investigación europea es la ausencia de diálogos con los enfoques críticos en comunicación popular, educativa, para el cambio social o decolonial de origen latinoamericano, un hecho evidente en el territorio anglosajón, italiano o germanoparlante, y en el que son excepcionales los países que actúan como puente o bisagra con respecto a sus vecinos de ultramar: Portugal con Brasil y, en especial, España con relación al resto de países hispanoparlantes. Sin embargo, al revisar muchas referencias

12 Desde estas publicaciones, también se ha intentado relacionar las distintas perspectivas que conforman el campo de lo ecológico y que, en buena medida, todavía permanecen fragmentadas y con un débil anclaje en teorizaciones clásicas. Nos referimos, por ejemplo, a la ecología de los medios desarrollada por autores como McLuhan y Postman, y otros enfoques más recientes como la information ecology de Nardi y O’ Day, el enfoque de las communicative ecologies (Tacchi et al.) y las media ecologies de Fuller, endeudadas con las herramientas teóricas de Guattari (Treré \& Mattoni, 2016; Treré, 2020). 
latinoamericanas comprobamos que el proceso de desconocimiento es de doble vía, dado que este territorio no siempre mira ni incorpora lo que producen sus homólogos del Norte.

En suma, si entendemos que el campo sigue manteniendo un rol periférico en comparación con áreas de estudio más exploradas, en lo siguiente su consolidación pasa por fortalecer puentes entre comunidades académicas que, hasta la fecha, parecen en exceso ensimismadas e incluso se caracterizan por una pretensión autofundante del campo. Esta perspectiva contribuiría, por otro lado, a "desoccidentalizar" los estudios comunicacionales (Curran y Park, 200o) y a percibir que existen rasgos comunes entre el activismo mediático clásico y el contemporáneo, o las luchas cada vez más emparentadas (en tanto conectan elementos materiales y postmateriales) y conectadas entre las comunidades del Norte y del Sur del planeta. En esta línea, apelamos a trabajos como los de Boaventura de Sousa Santos (2010), que intentan facilitar la interlocución entre comunidades académicas desde conceptos como los de "hermenéutica diatópica", que ponen el foco en la incompletud de toda cultura y en la necesidad de mejora a través del diálogo y la propia reivindicación de las epistemologías del Sur.

\section{Referencias bibliográficas}

Ardizzoni, M. (2009). Alternative media in Italy: The case of Telestreet. En M. Ardizzoni, \& C. Ferrari (Eds.). Beyond monopoly: Globalization and contemporary Italian media (pags. 171-184). Lanham, MD: Lexington Books.

Armstrong, D. (1981). A Trumpet to arms: Alternative Media in America. Cambridge, CA: South Press.

Askanius, T. (2020). Social movement studies and citizen media. En M. Bakert, B. Blaagaard, H. Jones, \& L. Pérez González (eds.), The Routledge Encyclopedia of Citizen Media. London: Routledge.

Askanius, T., \& Gustafsson, N. (2010). Mainstreaming the alternative: The changing media practices of protest movements. Interface: a journal for and about social movements, $2(2), 23-41$.

Atton, C. (2001). Alternative media. London, Thousands Oaks, CA: Routledge \& Sage.

Atton, C. (2004). Alternative Internet. Radical media, politics and creativity. Edinburg: Edinburgh University Press.

Atton, C. (2015). The Routledge companion to alternative and community media. New York: Routledge.

Badenes, D. (2020). Mapas para una historia intelectual de la comunicación popular Ideas, contextos y prácticas editoriales de los '6o y 'zo en América Latina. Tesis doctoral no publicada. Universidad Nacional de La Plata. http://sedici.unlp.edu.ar/handle/10915/103944

Bailey, O. G., Cammaerts, B., \& Carpentier, N. (2008). Understanding Alternative Media. Maidenhead: Open University Press.

Bakert, M., Blaagaard, B., Jones, H., \& Pérez-González, L. (Eds.) (2020). The Routledge Encyclopedia of Citizen Media. London: Routledge. 
Baldelli, P. (1977). Informazione e controinformazione. Milan: Baldolla.

Bakthin, M. (1965). Tvorchestvo Fransua Rable. Moscow: Khudozhestvennia Iiteratura. [Bajtin, M. (1998). La cultura popular en la Edad Media y en el Renacimiento. El contexto de François Rabelais. Madrid: Alianza].

Barassi, V. (2015). Activism on the web: Everyday struggles against digital capitalism. New York: Routledge.

Barranquero, A. (2015). Trayectorias, cruces y diálogos entre la tradición latinoamericana y anglosajona en comunicación comunitaria y ciudadana. Revista Internacional de Comunicación y Desarrollo (RICD), 1(1), 19-22. https://revistas.usc.gal/index.php/ricd/ article/view/2360

Barranquero, A. (2020). De-westernizing Alternative Media Studies: Latin American Versus Anglo-Saxon Approaches from a Comparative Communication Research Perspective. En J. Servaes (ed.), Handbook of Communication for Development and Social Change (329-340). Springer. https://doi.org/10.1007/978-981-10-7035-8_69-1

Barranquero, A., \& Treré, E. (2020). Los estudios sobre comunicación alternativa y comunitaria en Europa desde una perspectiva histórica y comparada. En F. Oliveira, G. Kaplún, M. Vicente, \& L. Custodio (eds.), Tradiciones de investigación en diálogo: Estudios sobre comunicación en américa latina y europa (pp. 201-224). Media XXI.

Benjamin, W. (1934). "El autor como productor". Conferencia dictada en el Instituto para el Estudio del Fascismo, 27 de abril. [(1998). "El autor como productor". En W. Benjamin. Tentativas sobre Brecht. Iluminaciones III. Madrid: Taurus].

Berardi Bifo, F. (2006). Skizomedia. Trent'anni di mediattivismo. Roma: DeriveApprodi.

Berardi Bifo, F., Jaquemet, M., \& Vitali, G. (2003). Telestreet. Macchina immaginativa non omologata. Milano: Baldini, Castoldi Dalai.

Berrigan, F. J. (Ed.) (1977). Access: Some Western models of community media. París: Unesco.

Brecht, B. (1927). Der rundfunk als kommunikationsapparat. Berliner Börsen-Courier, 25 diciembre. [(2006): The radio as an apparatus of communication (2-3). En A. Gumucio Dagron, \& T. Tufte (Eds.), Communication for social change anthology: Historical and contemporary readings. New Jersey, SO: Communication for Social Change Consortium, 2-5.]

Cammaerts, B., Mattoni, A., \& McCurdy, P. (Eds.) (2013). Mediation and protest movements. Bristol: Intellect.

Carpentier, N. (2011). Media and participation: A site of ideological-democratic struggle. Bristol: Intellect.

Carpentier, N. (2015). Community media as rhizome: Expanding the research agenda. Journal of Alternative and Community Media, 1, 4-6.

Carpentier, N., Lie, R., \& Servaes, J. (2003). Community media, muting the democratic media discourse? Continuum, 17(1): 51-68.

Cazenave, F. (1980). Les Radios libres: Des radios pirates aux radios locales privées. Que sais-je? Paris: P.U.F.

Cheval, J.J. (2013). "Guess who was on the radio last night?”. En G. Stachyra (Ed.), Radio. Community, challenges and aesthetics. Lublin, Polonia: Marie Curie \& Skłodowska University Press, 53-62.

Couldry, N. (2003). Beyond the Hall of Mirrors? En N. Couldry, \& J. Curran (Eds.), Contesting media power: Alternative media in a networked world. Lanham, Md: Rowman \& Littlefield Publishers, 39-56.

Couldry, N. (2004). Theorizing media as practice. Social Semiotics, 14, 115-132. 
Couldry, N. (2010). Why Voice Matters: Culture and Politics after Neoliberalism. Los Angeles, CA: Sage.

Couldry, N. (2012). Media, society, world: Social theory and digital media practice. London: Polity.

Coyer, K., Dowmunt, T., \& Fountain, A. (2007). The Alternative Media Handbook. London: Routledge.

Curran, J., \& Park, M. (Eds.) (200o). De-Westernizing Media Studies. London: Routledge.

Dalle, M. (2006). Les radios libres, utopie 'deleuzoguattarienne'. French Cultural Studies, 17(1), 55-72. https://doi.org/10.1177\%2Fo957155806060795

D' Arcy, J. (1969). El derecho humano a comunicar. Serie de Estudios y Documentos de Información, 36. París: Unesco.

Dark, S. (2009). Libere! L'epopea delle radio italiane degli anni settanta. Viterbo: Stampa Alternativa.

Debord, G. (1967). La société du spectacle. París: Buchet-Chastel. [(1999). La sociedad del espectáculo. Valencia: Pre-Textos].

De Certeau, M. (1980). L'invention du quotidien. Arts de faire. Vol. 1. Paris: Gallimard. [(1999). La invención de lo cotidiano. I Artes de Hacer. Ciudad de México: Universidad Iberoamericana].

Diani, M., \& McAdam, D. (Eds.). (2003). Social movements and networks: Relational approaches to collective action. New York, NJ: Oxford University Press.

Díaz Bordenave, J. (1977). Communication and rural development. Paris: Unesco.

Downing, J. (1984). Radical media: The political experience of alternative communication. Boston, MA: South End Press. [Reeditado como: Downing, John (2001). Radical media: Rebellious communication and social movements. London \& Thousand Oaks, CA: Thousand Oaks].

Downing, J. (2008). Social movement theories and alternative media: an evaluation and critique. Communication, Culture \& Critique, 1, 40-50.

Downing, J. (Ed.) (2010). Encyclopedia of social movement media. London: Sage.

Eco, U. (1968). La struttura assente: La ricerca semiotica e il metodo strutturale. Milano: Bompiani. [(1974). La estructura ausente. Introducción a la semiótica. Barcelona: Lumen].

Enghel, F., \& Noske-Turner, J. (eds.) (2018). Communication in international development: Doing good or looking good? London: Routledge.

Enzensberger, H. M. (1970). Constituents of a theory of the media. New Left Review, 64, Nov/ Dec, 13-36. [(1984). Elementos para una teoría de los medios de comunicación. Fragmentos. Barcelona: Anagrama].

Ferron, B. (2012). Les médias alternatifs: 'Contre-culture' ou 'sous-culture'? Les luttes de (dé) légitimation de la communication contestataire à travers l'étude de publications académiques et militantes. Tesis de doctorado no publicada. Universidad de Rennes 1.

Freire, P. (1973 [1969]). ¿Extensión o comunicación? La concientización en el medio rural. Ciudad de México: Siglo XXI.

Fuchs, C. (2010). Alternative media as critical media. European Journal of Social Theory, 13(2), 173-192.

Girard, B. (1992). A passion for radio: Radio Waves and Communities. Black Rose. [(1992). Radioapasionados. 21 experiencias de radio comunitaria en el mundo. Quito: CIESPAL].

Glessing, R.J. (1970). The underground press in America. Bloomington: Indiana University Press. 
Gómez García, R., \& Treré, E. (2014). The \#YoSoy132 movement and the struggle for media democratization in Mexico. Convergence. The International Journal of Research into New Media Technologies, 20(4), 496-510.

Gordon, J. (2008). Notions of community: A collection of community media debates and dilemmas. Oxford: Peter Lang.

Guattari, F. (1978). Les radios libres populaires. La Nouvelle Critique, 115: 77-79.

Gitlin, T. (1980). The whole world is watching. Berkey: University of California.

Gumucio, A. (2001). Making Waves. Stories of Participatory Communication for Social Change. New York: Rockefeller Foundation.

Gurevitch, J., Koricheva, J., Nakagawa, S., \& Stewart, G. (2018). Meta-analysis and the science of research synthesis. Nature, 555(7695), 175-182.

Habermas, J. (1962). Strükturwander der Öffenlichkeit. Hermann Luchterhand Verlag. [(1990). Historia y crítica de la opinión pública. La transformación estructural de la vida pública. Ciudad de México: Gili].

Halloran, J., Murdock, G., \& Elliott, P. (1970). Demonstrations and communication. A case study. Harmondsworth: Penguin.

Hamelink, C., \& Hoffmann, J. (2008). The State of the Right to Communicate. Global Media Journal, 7(13), 1-16.

Hepp, A., \& Couldry, N. (2009). What should comparative media research be comparing? Towards a transcultural approach to 'media cultures'. En D. K. Thussu (ed.), Internationalizing media studies (pp. 32-47). Londres: Routledge.

Howley, K. (Ed.) (2010). Understanding community media. Thousand Oaks, CA: Sage, 1-14.

Inglehart, R. (1977). The silent revolution:Changing values and political styles among Western publics. Princeton: Princeton University Press.

Jankowski, N. (2006). Creating community with media: History, theories and scientific investigations. En L. A. Lievrouw, \& S. Livingstone (Eds.), The handbook of new media (55-74). London: Sage.

Jankowski, N., Prehn, O., \& Stappers, J. (Eds.) (1992). The people's voice: local radio and television in Europe. London: John Libbey.

Kaplún, G. (2019). La comunicación alternativa entre lo digital y lo decolonial. Chasqui Revista Latinoamericana de Comunicación, 141, 75-94. https://doi.org/10.16921/chasqui. voi141.4077

Kidd, D. (2003). Indymedia.org: A New Communications Commons. En M. McCaughey \& M. D. Ayers (Eds.), Cyberactivism: online activism in theory and practice (pp. 47-69). New York: Routledge.

Kluitenberg, E. (2011). Legacies of tactical media. Amsterdam: Institute of Network Cultures.

Lefebvre, T. (2011). La bataille des radios libres: 1977-1981. París: Nouveau Monde.

Lewis, P. et al. (Ed.) (1984). Media for people in cities: a study of community media in the urban context. París: Unesco.

Lewis P. (Ed.) (1993). Alternative media: Linking global and local. París: Unesco [(1995) Medios de comunicación alternativos: La conexión de lo mundial con lo local. París: Unesco].

Lewis, P., \& Booth, J. (1989). The invisible medium: Public, commercial and community radio. Basingstoke: Macmillan Education.

Mahony, N., \& Stephansen, H. C. (2016). The 'frontiers' of participatory public engagement. European Journal of Cultural Studies, 19(6).

https://doi.org/10.1177\%2F1367549416632007

Manyozo, L. (2012). Media, communication and development. Three approaches. London: Sage. 
Martín-Barbero, J. (1987). De los medios a las mediaciones: comunicación, cultura y hegemonía. Barcelona: Gustavo Gili.

Mattelart, A. \& Siegelaub, S. (Eds.) (1979). Communication and Class Struggle. 2 vols. New York, NJ: International General.

Mattoni, A., \& Treré, E. (2014). Media practices, mediation processes, and mediatization in the study of social movements. Communication Theory, 24 (3), 252-271. https://doi. org/10.1111/comt.12038

Melucci, A. (1996). Challenging codes. Collective action in the information age. Cambridge: Cambridge University Press.

Mercea, D., Iannelli, L., \& Loader, B. D. (2015). Protest communication ecologies. Information, Communication \& Society, 19(2), 279-289. https://doi.org/10.1080/1369118X.2015.1109701

Milan, S. (2013). Social movements and their technologies: Wiring social change. London: Palgrave Macmillan.

Milan, S., \& Gutiérrez, M. (2015). Citizens' Media Meets big data: the Emergence of data activism. Mediaciones, 14, 120-133.

Park, R.E. (1922). The immigrant press and its control. New York: Harper.

Pasquinelli, M. (2002). Media activism: Strategie e pratiche della comunicazione indipendente. Roma: Derive Approdi.

Peppino Barale, A.M. (1999). Radio educativa, popular y comunitaria en América Latina. Origen, evolución y perspectivas. Ciudad de México: Plaza y Valdés.

Pérez Martínez, J.E. (2018). La radio libre en Madrid (1976-1989): los orígenes del movimiento por la libertad de emisión. Commons. Revista de Comunicación y Ciudadanía Digital, 7(1), 112-143. http://dx.doi.org/10.25267/COMMONS.2018.v7.i1.04.

Pertierra, A. C., \& Salazar, J. F. (Eds.). (2019). Media cultures in Latin America: key concepts and new debates. London: Routledge.

Plant, S. (1992). The most radical gesture: The situationist international in a postmodern age. London: Routledge.

Rennie, E. (2006). Community Media: a global introduction. Lanham, MD: Rowman \& Littlefield.

Reyes Matta, F. (ed.) (1983). Comunicación alternativa y búsquedas democráticas. Ciudad de México: ILET/Friedrich Ebert Stiftung.

Rodríguez, C. (2001). Fissures in the mediascape. An international study of citizen's media. Cresskill, NJ: Hampton Press.

Rodríguez, C., Ferron, B., \& Shamas, K. (2014). Four challenges in the field of alternative, radical and citizens' media research. Media, Culture \& Society, 36(2), 150-166. Recuperado de https://doi.org/10.1177\%2Fo163443714523877

Sandoval, M. (2009). A critical contribution to the foundations of alternative media studies. KurguOnline. International Journal of Communication Studies, Vol. 1.

Sandoval, M., \& Fuchs, C. (2009). Towards a critical theory of alternative media. Telematics and Informatics, 27(2), 141-150.

Santana, M., \& Carpentier, N. (2010). Mapping the rhizome. Organizational and informational networks of two Brussels alternative radio stations. Telematics and Informatics, 27(2), $162-176$.

Santos, B. de Sousa (2010). Epistemologías del sur. Ciudad de México: Siglo XXI.

Servaes, J. (1999) Communication for development: One world, Multiple cultures. Cresskill, NJ: Hampton Press. 
Simpson, M. (Ed.) (1986). Comunicación alternativa y cambio social. Ciudad de México: Premia.

Soley, L.C., \& Nichols J.C. (1986). Clandestine radio broadcasting: a study of revolutionary and counterrevolutionary electronic communication. Westport: Praeger.

Stephansen, H. C. (2013). Connecting the peripheries: networks, place and scale in the World Social Forum process. Journal of Postcolonial Writing, 49(5), 506-518.

Stephansen, H. C. (2016). Understanding citizen media as practice. En Baker, M. y Blaagaard, B. (Eds.), Citizen Media and Public Spaces: Diverse Expressions of Citizenship and Dissent. London: Routledge.

Stephansen, H. C., \& Treré, E. (2019). Citizen Media and Practice: Currents, Connections, Challenges. London, New York: Routledge.

Thomas, P. N. (2017). The contributions of Raymond Williams and EP Thompson to communication and social change theory and practice. European Journal of Communication, $32(5)$, 405-418. https://doi.org/10.1177\%2Fo267323117723968

Treré, E. (2012). Social movements as information ecologies: Exploring the coevolution of multiple Internet technologies for activism. International Journal of Communication, $6,2359-2377$.

Treré, E., \& Mattoni, A. (2016). Media ecologies and protest movements: main perspectives and key lessons. Information, Communication \& Society, 1-17.

Treré, E. (2020). Activismo Mediático Híbrido: Ecologías, Imaginarios, Algorítmos. Bogotá: Documento N. ${ }^{\circ}$ 16. Friedrich-Ebert-Stiftung FES (Fundación Friedrich Ebert)-C3. http:// library.fes.de/pdf-files/bueros/la-comunicacion/17279.pdf

Treré, E. (2019). Hybrid Media Activism: Ecologies, Imaginaries, Algorithms. London, New York: Routledge.

Tufte, T., \& Mefalopulos, P. (2009). Participatory communication: A practical guide. Washington D.C.: The World Bank.

Uldam, J., \& Askanius, T. (2013). Online civic cultures: debating climate change activism on YouTube. International Journal of Communication, 7, 1185-1204.

Vidal Beneyto, J. (Ed). (1979). Alternativas populares a las comunicaciones de masa. Madrid: Centro de Investigaciones Sociológicas. 
